\title{
The Concept of Leadership is Always Engaging for an Elite Occupational Preeminence
}

\author{
https://doi.org/10.21272/bel.5(2).139-146.2021
}

\author{
Dr. Rudrarup Gupta, ORCID: https://orcid.org/0000-0003-2961-1502
}

Sustainable Cosmos Ambassador, Global Change Maker and SDG Expert of Green ThinkerZ Society, CEO, Multifarious Projects Group, Kolkata, India

\begin{abstract}
The term "Management and Leadership" are the very prime focus for any of the very enriching occupational steps to deal with most significant progress. Most importantly, both education and occupation are largely dependent upon the same because the term "Management" shall be navigating the most legitimate avenues to accomplish the task with the finest objectives. On the other hand, the term "Leadership" shall be focusing upon the methodology and best future consequences respectively. It is quite evident that both academic leaders and organizational leaders shall have to focus for the vivid progressions along with the radiant dimension of success in terms of best financial outputs, occupational vibrancy, global appreciations and undeniable good will at all. This is the primary need of those leaders to refine both education and organization in a befitting manner. This is how they may enrich the best occupational goal with momentous dexterity, where economical solidity is one of the significant measures consequently. Therefore, leaders are those very eminent personalities, who are here to execute their best through the stature of prime competency for leading the hierarchy of an organization without any second thought because their elite motive to ensure the comprehensive organizational success and the most rapid celerity with best available resources, indeed. That is how leaders do empower their followers who shall have to be well trained not only for the self enrichment within the stipulated time frame but to contribute a lot for the unbeaten occupational culmination in a conforming manner. Leaders have been finding some of the very dynamic objects through some outstanding organizations like "Indian Statistical Institute". It a prime institute of "National Importance". It is India based research institute which consists of all the scientific research which are of absolute elite class indeed. All the academic leaders are comprehensively seeking for the comprehensive innovations along with their global clarities and those are going to be the historic discoveries for the best consequential research soon. All the forthcoming researchers will be undoubtedly successful, and they shall have the exclusive vive to accept that exemplary methodologies of research in the end. Therefore, their conceptual depth would really be high, and they shall be able to implement those core concepts like departmental culmination, communal advocacy, collective perception, proclamation of ideas, elevation of various convictions and the amalgamation of multifarious exposures will definitely be determined. On the other hand, renowned institute like ISI finds the captivating solutions for the researchers through their insightful data and their comprehensive analysis respectively. So, each researcher can have the best analytical review not only to focus our extensive splendor of research but to refine our collective benefaction over a due course of time.
\end{abstract}

Keywords: Educational Magnificence, Leadership Approaches, Organizational Furtherance Leaders' Contributions.

JEL Classification: L100, M100.

Cite as: Gupta, R. (2021). The Concept of Leadership is Always Engaging for an Elite Occupational Preeminence. Business Ethics and Leadership, 5(2), 139-146. http://doi.org/10.21272/bel.5(2).139-146.2021.

Received: 03 May 2021

Accepted: 12 June 2021

Published: 25 June 2021

Copyright: (C) 2021 by the author. Licensee Sumy State University, Ukraine. This article is an open access article distributed under the terms and conditions of the Creative Commons Attribution (CC BY) license (https://creativecommons.org/licenses/by/4.0/).

\section{Introduction}

Leaders do take the noteworthy initiatives to excel their absolute integrities, to achieve the largest organizational goals very shortly, which shall be very stimulating for both leaders and followers to inculcate some exceptional experiences, to ensure the best commercial dealing and the impressive financial outputs as per their most prestigious strategy. That is why they need the exclusive trainings and the sound exposures not only to enhance the best commercial profit margin but to expand the streamlined inclinations of that very organization and their first-rated multifaceted developments as well. This process does evaluate the utmost 
participation of their followers to be involved in that organization. It is quite needless to state that the concept of "Participative Leadership Approach" shall have to be instigated as soon as possible for the overall evaluation for both leaders and followers. Otherwise, leaders will not be able to discover the strengths and weaknesses from their immediate subordinates and followers. So, how shall it be possible to win the spirited occupational attainments right from the beginning? It is a process of establishing a family, where the entire official aspirations and the administrative challenges shall have to be consummated as a hole. Most importantly, followers from entire hierarchy shall have to raise their voices and valid points for their collective refinements right from knowledge to their felicitous executions at the end of the day. That is how, the great leadership transformation is possible by the most dynamic leaders because skillful organizational actualizations need the high-end educational competency, and it is one of the most pivotal issues in all the regards. That is the reason why leaders are desirous to bring out the real parity in between their standing of education and ardent knowledge and on the other hand, they must have the heartfelt realizations for the organizational spirit and need as well. Both the perspectives would be comprehensively evaluated by the leaders, and they shall disclose the final outcomes about their collective strengths of team right from the proper detection and the executions of elite class indeed.

This is how, the leadership transformation is correctly directed and entrenched by the leaders to ensure the high-end vertex, where educational competency and organizational purifications are undoubtedly possible and that is possible just within the stipulated time frame. According to the legitimate protocol of leaders, they should transform their followers to enhance the best skillful potentials not only to adopt the best leadership qualities but to convert their qualities in enriching the supreme performances in connection with the destined regulatory mission in a very equitable dimension for successful tomorrow. So, these are the possible profitmaking avenues to generate unique occupational propositions at all. That is the reason why leaders are solely responsible to implement their unequivocal plans to explore and they do play the sheet anchor role as eyeopeners for all the associate followers with intensified encouragements at the end. According to their sustainable objectives this is the finest root of segregated work force, where both leaders and followers shall have the equal opportunities to refine their exposed thoughts and the untold introspections as well. Zakeer Ahmed Khan, Dr. Allah Nawaz and Irfanullah Khan have expressed their most invaluable views in one of the published articles entitled: Leadership Theories and Styles: A Literature Review (2016) that, various explanations, classifications and definitions may enrich our contemporary literature indeed. If all the leadership styles are properly clarified, then both organizational and social research will be equally considered.

In the same light, the versatile contributions of transformational leaders will be impactful from the perspectives of both education and an organization not only in terms of glorious economic growth but for the imperative celerity to hold the profession in a legitimate style because each leader is really goal oriented and they do invariably focus upon performance and various evaluations. Therefore, followers of entire hierarchy are very conscious for the self assessments. Otherwise, the perfection of them will not be possible and on the contrary, the compact professional structure will be beyond the reach. In this regard, professional initiatives will have to be really enlarged for the most aristocratic benchmark and that will be really impactful for all the sustainable goals to achieve through the best cognizance of financial enhancement, educational merriment and the organizational achievement at the same point of time.

\section{Research Illustrations}

This is the illustrious concept and the best authoritative headway, where both leaders and followers and on the other hand, all the students and the teachers should equally be confined to enact for the forthcoming enterprises to focus upon and to set the significant instances in the history. Therefore, leaders are such well-wishers and great path influencers, who are aiming for the very specific avenues to deal with. The following points are very crucial in this regard.

They should have the specific intention for the collective professional solace, where financial strength, their communal existence and the unparallel value of positivity are absolutely stunning to move to. That is why leaders do need the empirical study, which signifies both transformational and transactional approaches of leadership for their comprehensive automation of competent milestones to reach lawfully.

Leaders need the worthwhile accession to enhance their thoughts and envisions in a global platform because their irreplaceable global efforts and initiatives will be definitely very attractive to adopt the root of professional exhilarations, where each follower and employee shall have the exclusive charm and they will be able to satisfy their respective discipline not only for the societal entrance but for the optimistic outcomes also. Leaders do prefer the flawless globalization. As a result, they will be able to cope up the best global paradigm 
and the active occupational diligence, respectively. This is the refining business procurement to the welldeserved professional community. The captivating bonding between leaders and followers for reinforcing each and other to win this magnificent battle of teamwork and culture of inhabitable learning.

Apart from the same, another most exemplary attention should be the network. As a reflection it would be both education and occupation which will definitely be in an indispensable focus of proficient culmination. Leaders do have the endurable responsibility to clarify the florid desire of our learners from the end of both education and organization. It is endless in nature and in case of rapid refinements the entire global network will be truly supporting to those leaders to utilize all the needful opportunities as a hole. In this regard leaders should be communicating with all the other overseas leaders to collaborate and to ensure the best commercial assignments at the end of the day. Therefore, all the learners will be really courageous not only to develop their collective learning but to generate the highest occupational perceptions through the effect of flawless communication throughout the year. The key idea is all the scholar students are having some inextinguishable scopes to study abroad and they are trying to accomplish their great educational height from there and they are being invariably involved to deal with the international business of absolute elite class. The author thinks they shall fulfill both the avenues of education and profession under the sublime guidelines of global leaders to reach at the pick soon.

$>$ In the same light, leaders do concentrate upon the operation as well. Because any successful operational flow is invariably necessitous not only to extract the best performance from the employees but to pay the decisive courage to the students. Leaders are trying to pay the real equality to the entire society between all the mentors and mentees and in other words, all the leaders and their well-deserved subordinates as well. It is a real balance, and it may reform the most unequivocal future consequences very minutely.

Therefore, leaders are prepared and trained very magnificently to find two masterly boulevards of both education and occupation simultaneously. Rose Ngozi Amanchukwu, Gloria Jones Stanley and Nwachukwu Prince Ololube have deciphered their collective thoughts in one of the published articles entitled: A Review of Leadership Theories, Principles and Styles and their relevance of Educational Management (2015) that, nation cannot be grown up without the influence of educational leaders. The prime focus of this research manuscript is to determine the wider concept of leadership and the impacts of school management.

\section{Methodology}

It is an exclusive research abstract, which has already been composed through self-cognitive practice based upon the pure research focus of exclusive theory, where leaders and followers are always in the same platform to frame the most mutually exclusive bonding to make sure that, they are undoubtedly participative in nature to understand about their forthcoming communal responsibilities in terms of educational acceleration and the organizational inventiveness. Both the chapters are invariably interconnected with each and other to find out the avenue of common victory of sustainability in enriching their real economic growth, communal magnificence, and the brand value reach of academic and organizational leaders indeed. It comprises pretraining method, learning and post learning consequently. That is why, they have entrenched their considerable focus upon the following:

\section{Pre-Training Method}

$>$ Rigorous Training: Leaders are liable to pay the needful training for the rapid growth according to their all-round parameters. So that it is possible to take all the mentees and followers to enhance their collective determinations. In this respect, they will be able to generate the additional trust upon themselves to work hard for our genuine stimulations. They shall be reflecting their depth of education and learning for the competent shape of an organization without any second thought. This is how both education and the implementation of learning may influence upon the best organizational greatness along with the magical consequence of our national legacy and the vigilant summery of success.

$>$ All-round Motivation: According to my analytical perception we need the motivation at every individual stage of our lives at all. So that our overall competency will be functioning proper for the wide-ranging accuracy. Both competency and accuracy are very indispensible for an organization. As a matter of the fact is leaders are solely responsible to motivate their students, subordinates and followers to fulfill their utmost professional target within the stipulated time frame. It is an interesting initiative which is allotted to the leaders to adopt the process and to devote for the discreet professional mission through the best educational core.

$>$ Context of Goal: Leaders do wish to reach the target along with their great team members who are not only ambitious but to display the best all-round performance to enrich the goal. Leaders are very adoptive for 
assignments and multifarious tasks to focus upon. They firmly believe that bright organizational ease means the invaluable reflection of education. So educational values are the prime focus from the point of view of leaders in enriching their self-erudition which is accordingly reflective for professional enlightenment. They do train their followers as per the concept of advocacy leadership approaches and they do expect for the diligent occupational glory.

Learning. It is a real time to move for the pre-scheduled betterment upon the past experiences. Leaders do think about the future and that is the reason why they do ensure the comfortable presence on the basis of past for sustaining in the highly competitive future. So, it is a big deal for both leaders and followers to participate in connection with the delightful occupational discovery, where the commercial benefits are just a matter of time.

Publicity: It is indeed very crucial in this methodology. Most importantly, the author has invented the most profit-making discovery, where people shall have the rousing opportunities to publicize their collective vision and the exemplary hard works at the end. On the other hand, it should bring out the inhabitable parity between the desire and the flow of execution consecutively. It is very helpful to ensure the balance between bodies and minds as well. So that, it is quite evident for the leaders to secure the psychological harmony for themselves. It is a promising step to prepare the followers who may come in front with never-ending professional dynamism from all the undesirable resilience.

$>$ Implementation of Scopes: Leaders do search for the variety of scopes all the time because it is highly demanding for all our followers to cherish the most glistening objectives with positivity. Therefore, it is undoubtedly very magnetic and compelling for the leaders to stimulate their mentees and followers to walk upon the same track to crack the historic visionary because without scopes hopes will not be coming in front. As a result, both leaders and followers will not be able to explore the thoughts at all. Therefore, the methodology says that all the leaders shall have to be perceptual to bring out their best occupational manpower for accomplishing the said implemented scopes without any fail.

Cognitive Competence: Leaders do need the same to regulate the real organizational paradigm along with the exclusive brilliance indeed. This cognition might be the unavoidable medium to think upon the all-round developments so that both education and organization might be in the same track to be evaluated. Most notably the prime importance is to have the sound education. It shall be undoubtedly proactive to join in an organization through the most impressive occupational perspicacity. That is why, both cognition and competence shall have to be under consideration to change the shape of our overall system and every individual leader and follower shall have to abide by the same. Then only this entire global academic uncertainty will be not here over the coming years and leaders shall have to remember that, without knowledge, education and the commendable conception organization cannot run, and it is never possible for them to accomplish the best organizational goal indeed.

Scrutiny of Need: It has the exclusive occupational essence because each leader and follower shall have to understand the nature and the absolute impulse of an organization. It is very important for him/her to be involved in an organization to match their most constructive perceptions with the same. That is how they are keen to direct the entire organization from the perspective of its fascinating occupational transparency to its congratulatory brilliance at the same point of time.

Post Learning. It is another very important aspect, where past experiences and the presently available resources are equally very important for the leaders to estimate the future. In this concept, they have decided to specify certain parameters for certifying the forthcoming assignments certainly.

Qualitative Measure: Everyone is incepted in this earth with qualities and those shall have to be really enriched through their significant refinements indeed. Without the aforesaid measure they will not be able to jump in these most prestigious occupational ventures along with their sound participations and learning as well. It is quite evident to share that the organizational practices are very delicate, and it is not so easy. Hence, it is indeed very essential for them to speculate about the best possible educational opportunities, which shall be undoubtedly very purposeful to grab the needful learning and that shall have to be implemented in their profession as well.

$>$ Exclusive Insights: Each leader is very worried about their conceptual insights because the entire development of an organization needs the utmost support of the leaders' right from the beginning. That is why, they must remember about the best reflection of education for the great professional zeal to ensure. Now they should be thinking about the thought process to drive the same for organizational prosperity through the 
unbeaten benefaction of learning and educational excellence at all. That is why, both leaders and followers should have the same intensity of mind not only to proceed further in terms of money and best financial outcome but it shall be the sound proposition of emphatic organizational virtuoso invariably.

$>$ Future Estimation: It means the best conceptual assessment and it is the inhabitable responsibility of our leaders to do the best analysis about the mentioned estimation. It is preferable to have the assumption in connection with the possible future consequences. Thus, they shall be arranging their required finance, best and competent manpower, significant professional planning, scopes of great decision making and so on. Therefore, it shall be undoubtedly mesmerizing for both leaders and followers to understand the market research and it is much needed to judge the situation for upcoming possibilities to enrich the superior organizational trend indeed. That is why, trained and experienced leaders should immediately be evaluated and appointed for this exclusive research to accomplish for the consequential future goals which are definitely desirable for them to sustain with anticipated occupational profit and best resource of financial outputs at the same point of time.

$>$ Predilection of Knowledge: It is very riveting, and it has the far-reaching impacts for the sparkling occupational speed of movements. That is why the foremost stage is to impart knowledge with exhilarating education at all. That is the spirited avenue to participate for combative occupational floor. It will be really meticulous for them to tackle various occupational uncertainties and those will be explicitly verified by the leaders for proceeding in the next step shortly. It is invariably true that education can bring out the strong metamorphosis with the profanities and positive propensities to project the classic vision of any organization.

The fact is that I have highlighted self-explanatory methodology from the perspective of leaders to enrich both the discipline of education and organization in terms of acceptance, accelerations and superiority. Most notably, leaders should be concentrating upon both the said disciplines to bring out the hope and scope to explore. This is what I have expounded in my research thesis along with the said form of knowledge from my heart-felt perception and belief. Furthermore, I really feel very crucial that they who are today's managers they are tomorrow's leaders. So effective leadership skills are vastly depending upon the self vision and the upright estimation as well. Therefore, the author has incorporated below some of the valid contexts to highlight my research focus which are as follows:

> Positive Vision: Leaders' brains are small but significant and far-reaching. Leaders do have the invariable vision that how far they will be able to run and how far an organization is able to grow through attaining profits and competent business goodwill. Leaders do frame the requisite channel accordingly. It is quite true that this noble vision is unquestionably instigated from substantial education, confidence and self desire respectively.

$>$ Mutual Stimulation: It is really very expensive in our human life. As a result, leaders do need the same throughout their best professional life to prosper in a very befitting manner. That is why leaders and followers do stimulate themselves with each and other. So, they do expect everybody to be under the sublime guidelines not only for collective learning with sound education but to grip the fundamental equation of their respective organizations as a hole. Stimulation means an additional zeal to store and the scope of rapid execution without any hesitation indeed. That means the leaders do intend for accomplishing thoughts to prevail in all over the globe. It indicates the alluring knowledge and learning which is undoubtedly required to entrench their best professional contemplations without any second thought. Leaders are the great motivators through their convincing style already. That is the reason why all the followers are in a very prior position to enhance the spirit for hard works and noticeable dedications. It is very natural for the leaders to extract the best output at the end. This is how the concept of "Stimulation" is established and it is really enriching for both the leaders and followers to excel their mutual stimulation.

$>$ Mentoring: The open-eyed fact is that leaders are the best path finders in all over the globe. They do nourish their deployed employees in such a manner that, employees do gain the enormous knowledge regarding their allotted fields, and they are in a commendable position to implement the same for well-deserved economical revenue.

Jyoti and Dr. Farhat Mohsin have contributed their high-end cognitive reflections in one of the published articles entitled: Current Practices and Challenges of Performance Management System in Higher Education Institutions (2020), that according to the present-day scenario most of the organizations have adopted the most valuable "Performance Management System". The objective is to refine the concept of "Human Resource". It is one of the noticeable organizational steps for the entire managerial hierarchy to concentrate upon their organizational and individual advancements indeed. Therefore, according to my research thesis I should be including three very expensive conditions for the leaders to certify my research focus. 
1. Leaders have to train their employee: It means the concept of "Advocacy Leadership Approach" which is definitely very impressive in this regard because all the employees do join in an organization after the completion of learning. Nevertheless, learning does not have specific end at all. Once the theoretical learning is finished the occupational learning is instigated. Apart from the bookish knowledge the efforts for occupational learning will be in vein. All the bookish conceptions should reflect about the strength of their objectivity. So, both the academic learning and occupational learning must match with each and other. It shall be really prevailing for the leaders to include the utmost strengths of their followers. It is quite evident that, academic learning helps and stimulates to participate in occupational learning. It means that, the primary learning is the base of secondary learning indeed. So, leaders are trying to enrich their both the notable vision and base of learning for multifaceted outputs to enhance the global flow of economy.

2. Leaders have to take the proper decision: Decision making is one of the elite procedures to have the best options out of various alternatives. We, the rational human being does take a number of decisions in our dayto-day life, but business decisions are always very specific and goal oriented. That is why, leaders do have the same in connection with the inhabitable progression of their organizations indeed. Therefore, it is a very important step for the leaders to search the best alternatives for solving any problem within the blink of an eye. It is absolutely very supportive to reach the best organizational goal with the utmost professional clarity. It means a lot for the followers to have the great vicinity of learning and prosperity along with their significant promotions and best financial sustainability. Therefore, this fascinating concept makes the exclusive bonding between leaders and followers and to accelerate their prevalent occupational goal to achieve and they shall definitely be able to motivate their next generations to maintain the same prestigious tradition for enabling the effective managerial performance with spirits.

3. Leaders have to reach the organizational goal: It is the ultimate factor and that is the reason why both leaders and followers are emphasizing the same. The fact is that they do place their collective decisions in a very conforming manner so that each individual can have the positive scope to participate, and they can convert their decisions to productive actions indeed. It shall be worth while once they will be well groomed, matured and prospective in nature so that it shall be very easier for them to feel the need of organizational goal, which is equally very important for them to learn the process of enrichment and it shall definitely in their favor to develop their course of actions and on the other hand detection of problems alongside the perceptual analysis. It is needless to convey that organizational goal needs the all-round eminence and meticulous paradigm and these are conceptually regulated by the leaders as well. So, it is very essential for the leaders to secure their advantageous educational core and mutual understanding. I believe, leaders shall have to take the invaluable initiatives to make it happen and it will be a grand occupational benchmark for all of us to earn our bread and butter at the end.

\section{Findings}

This research focus reflects the satisfying objectives of professional attainments where both leaders and followers are the best eye-openers to channelize the divine occupational vision, while they are very introspective, impactful and demonstrative towards their trained professional shape for all. So according to my most candid perception, leaders are the most imperial personalities who have found the solutions through the five tenets of life, and it is really influential for them not only to maintain their impetus fortitude but to glitter their omnipotent professional majesty in a very conforming manner. These five tenets are presented below:

Self Objectivity: It is one of the illustrious factors, where each leader shall possess for both learning of students and exploration of employees. It is a price less dimension for them to abide by the noble instructions of leaders but to make it realistic according to their progressive dynamism at all. It increases spirit and devotion to complete the assigned tasks very smartly. That is the reason why both leaders and followers shall have to have this studentship for refining their untold learning with momentous elevation of life indeed.

Self Practicability: It is highly spirited and thoroughly distinctive to enhance the desire of moral contributions. It is highly effective for our leaders to enrich their psychological dreams for both education and profession. Most importantly, it is very costly for self discovery and to know about the self strengths and weaknesses. Therefore, leaders do generate their actual self for their ardent knowledge and to disseminate the collective learning at the end. Without educational richness it is not possible, and leaders are the genuine generator for the explosive boulevard of knowledge for elevating their speed and it is simply the magnificent journey for real organizational goal.

Self Celerity: It creates the hope to reach the destiny. It is the ultimatum to snatch the best occupational victory as well. Leaders do generate the confidence and they aspire for their consequential milestone 
accordingly. Therefore, they need to have the unavoidable education and it is perceptually very effective for leaders and followers not only to run a successful organization but to preserve this exceptional momentum in the better consequential life. So, it is very important for students, who are going to be the most prestigious leaders across the globe with phenomenal exorbitance.

Self Originality: It signifies the present standing in a befitting manner. Academic leaders do guide their students to analyze the best qualitative measure of a student. Most importantly, it is really costly for them to focus upon this throughout the year so that it shall be as advanced as it is really expected not only to find the best academic excellence, but it shall be undoubtedly praiseworthy to convert the same in enriching their professional endeavor.

Self Viability: It is already accustomed by the leaders and that is the reason why leaders do include their followers who have already been proficient through their first-rated educational grandeur. It shall be very easier for them to demand for the noticeable occupational inventiveness, and it will be favorable for them to drag the organization for the fabulous global interpretations. So, it shall be the dashing intentional core and it is perpetually regulated by the leaders with gorgeous perspicacity of learning.

Busse Ronald has expounded his significant perceptions in one of the renowned published articles entitled: Comprehensive Leadership Review, Literature, Theories and Research (2014), that leadership has been comprehensively perceived in this manuscript. Most importantly author has described about the historical foundation of leadership after that modern leadership approaches in a most conforming manner. Therefore, all the leaders must be having the said propensities to research for an organizational glory where both educational dream and occupational zeal will be contributing together not only for their grand sustainability in all over the globe but to set the refulgent example for both histrionic and scientific discoveries in terms of imperishable financial affluence, immortal gesture of collective professional fame and most notably communal victory with graceful visionary.

\section{Conclusion}

This research focus is having the booming concept of multifaceted acquirements where both the concept of education and profession are well bonded to fabricate an enduring professional avenue with infinite hope to prosper soon. Therefore, I have penetrated the concept of "Leader" through the following:

1. L-Learning as per your best: It ensures the passionate magnificence of learning for entrenching their florid betterments to survive in this highly competitive globe.

2. E-Emerging originations: It shall be conducive for both leaders and their desired beginners to reach their occupational goals collectively with the extensive expansions of knowledge and spirited accelerations of quality.

3. A-Aspired demonstrations: An organization gives the incomparable stand of scopes to flourish. So leaders would be truly sensitized to adopt the education and evolve the profession at the same point of time so that the equality of transformation will definitely be reflected.

4. D-Destined magnitude: Leaders shall be emphasizing upon the same not only to showcase their occupational fame but to embrace their global omnipotence in terms of best commercial decree, global reformations and unblemished communal eminence.

5. E-Enduring solidity: The entire organization does incorporate this unique conception to draw the best attention because it is the enlightening spark for their all-round manifestation in connection with global revenue generation.

6. R-Realignment of stand: It is definitely possible for all the leaders and followers through their united cultural synthesis where the collective notion of learning will be invested and inquisitive professional goal will definitely be innovated since both educational zeal and occupational deal are perpetually interconnected for the reactive success forever.

\section{"Leadership is to realize the need and to visualize the success".}

Funding. There is no funding for this research. 


\section{References}

1. Khan Zakeer Ahmed, Nawaz Dr. Allah and Khan Irfanullah (2016). Leadership Theories and Styles: A Literature Review. Journal of Resources Development and Management, 16, 1-7. Available at: [Link]

2. Amanchukwu Rose Ngozi, Stanley Gloria Jones and Ololube Nwachukwu Prince (2015). A Review of Leadership Theories, Principles and Styles and their relevance of Educational Management. Management, 5(1), 6-14. [CrossRef]

3. Jyoti and Mohsin Dr. Farhat (2020). Current Practices and Challenges of Performance Management System in Higher Education Institutions. Journal of Critical Reviews, 7(7), 921-925. [Google Scholar]

4. Ronald Busse (2014). Comprehensive Leadership Review, Literature, Theories and Research, Advances in Management, 7(5), 52-66. Available at: [Link]

5. Regina Andriukaitiene, Valentyna Voronkova, Olga Kyvliuk, Marina Maksimenyuk and Aita Sakun (2017). Theoretical insights into expression of leadership competencies in the process of management. Problems and Perspectives in Management, 15(1-1), 220-226. [CrossRef]

6. Suzanne T. Koty (2020). Developing Teacher Leaders Through Professional Development Offered in a District Teacher Forum. Walden Dissertations and Doctoral Studies. Available at: [Link]

7. Shagranda M. Traveler (2019). Retention Strategies to Prepare and Maintain Talent for Future Leadership Roles. Walden Dissertations and Doctoral Studies. Available at: [Link]

8. David R. Kolzow (2014). Leading from within: Building Organizational Leadership Capacity. [Google Scholar]

9. European Agency for Special Needs and Inclusive Education, 2017. Inclusive Early Childhood Education: Literature Review. (F. Bellour, P. Bartolo and M. Kyriazopoulou, eds.). Odense, Denmark. Available at: [Link].

10.United Nations. Transforming our world: the 2030 agenda for sustainable development. Available at: [Link].

11.Stacy Marshall (2018). Followership and Leadership. Understanding Followership to Build Leadership. Virginia Polytechnic Institute and State University. [Google Scholar]

12.Brodie, Victoria Kimball (2019). Disrupted leadership: strategies and practices of leaders in a VUCA world. Theses and Dissertations. [Google Scholar]

13.Emily R. Lai (2011). Motivation: A Literature Review. Research Report. [Google Scholar]

14. Khushi Samina (2017). Strategic Planning for NGOs: A guide to understand the basics of strategic planning. Available at: [Link]

15.Nicholas Campion (2017). The Importance of Cosmology in Culture: Contexts and Consequences. In Trends in Modern Cosmology. [Google Scholar]

16.The U.S. Chamber of Commerce Foundation (2012). The Millennial Generation Research Review. Available at: [Link] 